\title{
NEW ANTENNAS AND METHODS FOR THE LOW FREQUENCY STELLAR AND PLANETARY RADIO ASTRONOMY
}

\author{
A. A. Konovalenko*, I. S. Falkovich*, H. O. Rucker ${ }^{\dagger}$, A. Lecacheux ${ }^{\ddagger}$, \\ Ph. Zarka ${ }^{\ddagger}$, V. L. Koliadin*, V. V. Zakharenko*, A. A. Stanislavsky*, \\ V. N. Melnik*, G. V. Litvinenko*, A. A. Gridin*, I. N. Bubnov*, \\ N. N. Kalinichenko*, A. P. Reznik*, M. A. Sidorchuk*, S. V. Stepkin*, \\ D. V. Mukha*, V.S. Nikolajenko*, R. Karlsson ${ }^{\dagger}$, and B. Thide ${ }^{\S}$
}

\begin{abstract}
According to the special Program of the National Academy of Sciences of Ukraine, creation of the new giant Ukrainian radio telescope (GURT) was started a few years ago on the UTR-2 radio telescope observatory. The main goal is to reach maximum band at the lowest frequencies (10-70 MHz), effective area (step-by-step up to 100,000 sq.m), and high interference immunity for resolving many astrophysical tasks when the sensitivity is less limited by the confusion effects. These tasks include stellar radio astronomy (the Sun, solar wind, flare stars, pulsars, transients) and planetary one (Jupiter, planetary lightnings, Earth ionosphere, the Moon, exoplanets). This array should be complementary to the LOFAR, E-LOFAR systems. The first stages of the GURT ( $6 \times 25$ cross-dipole active elements) and broad-band digital registration of the impulsive and sporadic events were tested in comparison with the existing largest decameter array UTR-2.
\end{abstract}

\section{Introduction}

Over many decades the basic interest of ground-based low-frequency radio astronomy in the field of star and planetary science was devoted to the radio emission of the Sun and the Jupiter. At the same time the advance of antenna assembly and digital appliances, as well as impressive projects of modern radio astronomy proposing a creation of a new

\footnotetext{
* Institute of Radio Astronomy, Ukrainian Academy of Sciences, Chervonopraporna 4, 61002, Kharkiv, Ukraine

† Space Research Institute, Austrian Academy of Sciences, Schmiedlstrasse 6, A-8042 Graz, Austria

‡ LESIA, Observatoire de Paris, UMR CNRS 8109, 92195 Meudon, France

$\S$ Swedish Institute of Space Physics, SE-751 21 Uppsala, Sweden
} 
antenna generation, permit ones essentially to extend a circle of investigated problems. At the present work this will be illustrated by the application of the (largest in the world) UTR-2 radio telescope which is at a complex modernization state during the last time. The application of some generations of progressively-improved receive-registering devices, observation methods distinctly increased the efficiency of the research of many objects in the Galaxy and the Metagalaxy, in particular, star and planets. Peculiar perspectives in this trend of radio astronomy will appear after the implementation of new radio telescopes LOFAR (The Netherlands), E-LOFAR (Europe), LWA (USA), LSS(France) and GURT (Ukraine).

\section{The Tasks of the Ground-based Low Frequency Radio As- tronomy (10-100 MHz) in the Stellar and Planetary Science}

In the case when the instruments for LF radio astronomy provide high values of sensitivity, dynamic range, interference immunity, frequency, time and space resolutions, the list of objects of different types in the Solar system and Galaxy which are opened for investigations is greatly widened (Table 1). In spite of the various physical natures of these objects, the mechanisms of the generation are similar in many aspects. This radio emission arises mostly when waves and charged particle beams are moving in magnetically active plasma. This leads to very intensive and in some cases coherent emission especially at the extremely low frequencies. Investigations of the Solar-Earth relations (space weather) are of special interest. Similar tasks can be formulated when considering stellar-planetary relations. Of course, it is interesting for the low frequency radio astronomy to study active (flare) stars and exoplanets (hot jupiters). Pulsars and, possibly, objects with transient emission are among stellar population. As well, the search for CII regions near type $\mathrm{B}$ stars using carbon radio recombination lines (similarly to the HII regions near O stars) is promising for the stellar astronomy.

It is necessary to emphasize that the listed opportunities follow not only from theoretical estimations but they are also confirmed by the numerous real experiments at UTR-2 that are described below.

\section{Existing Radio Telescopes and Instrumentation}

The radio telescope UTR-2 still remains the largest decameter instrument: frequency range $8-32 \mathrm{MHz}$, size $2 \times 1 \mathrm{~km}$ - T-shape configuration, maximal effective area 150,000 sq.m. [Braude et al., 1978; Konovalenko, 2000]. The telescope is equipped with a broadband system of antenna amplification which is immune to interference [Abranin et al., 2001]. A great number of research programs is carried out using the telescope aimed at investigation of almost all types of objects in the Universe, including planets and stars.

The effective use of the radio telescope would be impossible without high-quality backends. 12 years ago, a digital spectral processor DSP was installed at UTR-2. The device was developed in Austria and France; it was the first broad-band digital receiver for LF 
Table 1: The tasks of the ground-based low frequency radio astronomy (10-100 MHz) in the stellar and planetary science

\begin{tabular}{|l|l|}
\hline \hline & \multicolumn{1}{|c|}{ Solar system: } \\
\hline 1. & The Sun (sporadic radio emission) \\
2. & The Sun (quiet) \\
3. & Solar wind (IPS, scattering) \\
4. & Earth ionosphere \\
5. & Space weather \\
6. & Jupiter (DAM) \\
7. & Planetary lightnings (Saturn, etc.) \\
8. & The Moon (cosmic rays) \\
\hline & \multicolumn{1}{c}{ Galaxy: } \\
\hline 9. & Exoplanets (hot jupiters) \\
10. & Active stars \\
11. & "Galactic" weather \\
12. & Other stars (i.e. C II regions near B-stars, RRL's) \\
13. & Pulsars \\
14. & Transients \\
\hline \hline
\end{tabular}

radio astronomy with a real time Fourier spectral analyzer [Lecacheux et al., 1998]. Parameters of the DSP receiver and the next version (ROBIN) are shown in Table 2. A great amount of observations were performed during 1997-2007 using these two receivers, that yield a broad range of new results the Sun, Jupiter, pulsars, interplanetary scintillations, radio sources of continuum [Lecacheux et al., 2004].

The progress in computer and digital technology made it possible to create a new generation of receivers for UTR-2 (named DSP-Z) with performance superior to the predecessors [Kozhin et al., 2007; Ryabov et al., 2010]; the parameters are shown in Table 2. Two receivers were developed and manufactured within the Exoplanet ANR project (project leader Ph.Zarka); 5 receivers - within the Special Programme of NASU (A.Konovalenko). At present, 5 new receivers are installed at UTR-2 (to support its 5-beams operation mode), and two receivers - at URAN-2 and URAN-3 antennas.

The 5-beams mode of UTR-2 ensures maximal flexibility and efficiency of research: wide field of view (approx. 2 degrees), the possibility to control ionosphere refraction and to organize ON-OFF operation modes. All 5 devices can operate synchronously, to measure auto-spectra, complex cross-spectra for single- and multi-antenna modes, as well as to carry wave-form registration.

The current combination of the parameters of UTR-2 and back-end facilities meets requirements of stellar and planetary radio astronomy for frequencies below $30 \mathrm{MHz}$. 
Table 2: Broad-band real-time FFT receivers for the low frequency radio astronomy

\begin{tabular}{|l|c|c|c|}
\hline \hline Parameters & $\begin{array}{c}\text { DSP } \\
\text { (DSP microchips) }\end{array}$ & $\begin{array}{c}\text { ROBIN } \\
\text { (FPGA technology) }\end{array}$ & $\begin{array}{c}\text { DSP-Z } \\
\text { (FPGA techn.) }\end{array}$ \\
\hline \hline Frequency band [MHz] & 12.5 & 14 & 33 \\
\hline Number of freq. channels & 1024 & 2048 & 8192 \\
\hline Frequency resolution [KHz] & 12.0 & 7.0 & 4.0 \\
\hline Max. time resolution [ms] & 1.0 & 1.0 & 0.5 \\
\hline ADC resolution [bits] & 12 & 12 & 16 \\
\hline Dynamic range [dB] & 75 & 75 & 90 \\
\hline Input channels & 2 & 2 & 2 \\
\hline Auto- and cross-spectra & yes & yes & yes \\
\hline Wave-form & yes & yes & yes \\
\hline \hline
\end{tabular}

\section{Some New Examples of the Sun, Stellar, and Planetary Ob- servations}

\subsection{The Sun}

The flexible configuration of UTR-2 radio telescope and the potential of new equipment provide the opportunity for new operation modes. The use of two baselines with three antennas of the radio telescope and determination of the amplitude and phase crossspectra makes it possible to increase the reliability of identification of various effects in noisy conditions and to improve the precision of measurements of coordinates for various radio sources, in particular, in solar corona.

During recent years, a great amount of new data have been obtained at UTR-2 using the DSP receivers, including solar bursts of various types and detection of fine spectral and temporal structures [Melnik et al., 2004; Konovalenko et al., 2007].

Some dynamic spectra of sporadic solar radio emission obtained using three-antenna operation mode and DSP-Z receiver are shown at Figure 1.

\subsection{Interplanetary and Ionospheric Scintillations}

The observations of interplanetary scintillations at low frequencies give an unique possibility of outer solar corona diagnostics [Konovalenko et al., 2005]. Together with observations of solar bursts, the scattered radiation through the solar wind and ionospheric effects they give an useful information for the problem solutions of cosmic weather.

Dynamic spectra for 3C144 observations are shown at Fig. 2 (left). The interplanetary scintillations (vertical lines) are seen, and the scintillation index and scintillation spectra hold the information about solar wind parameters as well as unusual ionospheric scintillations with a complicated frequency-time structure. 

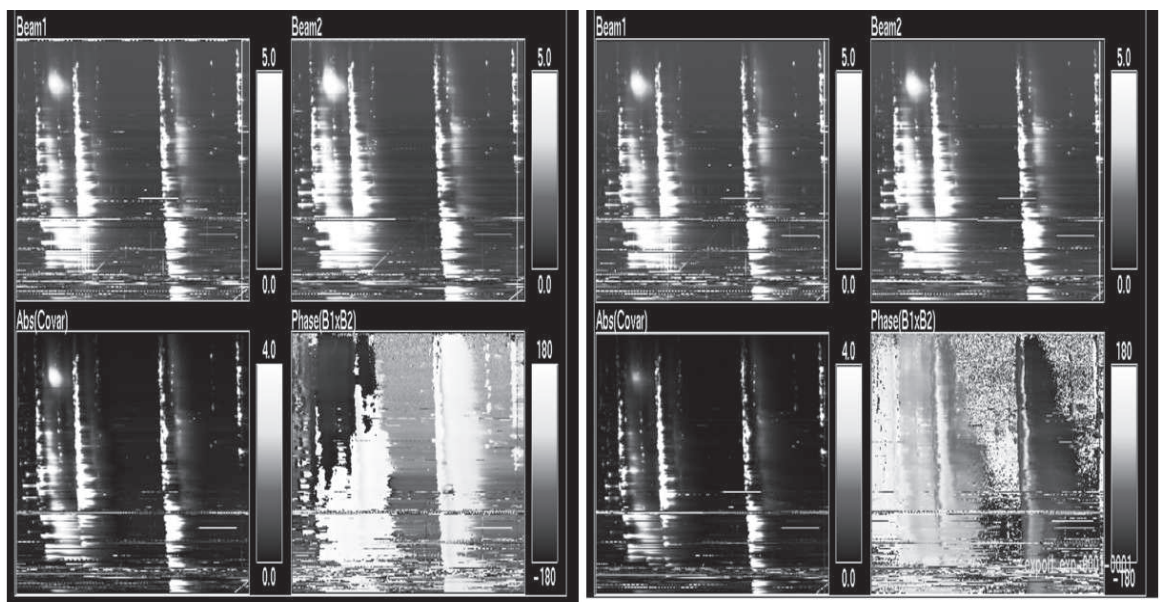

Figure 1: Solar bursts registered in 2-baselines operation mode. Dynamic spectra for pairs of antennas "West-North" (left) and "West-South" (right); the furthest halves of each antenna are used to increase angular sensitivity. The horizontal axis corresponds to 2 minutes interval, the verical one - to the frequency range 16.5 .. 33.0M Hz. Each set of 4 spectra comprises: the power for two antennas (top), the amplitude and the phase of cross-correlation (bottom).
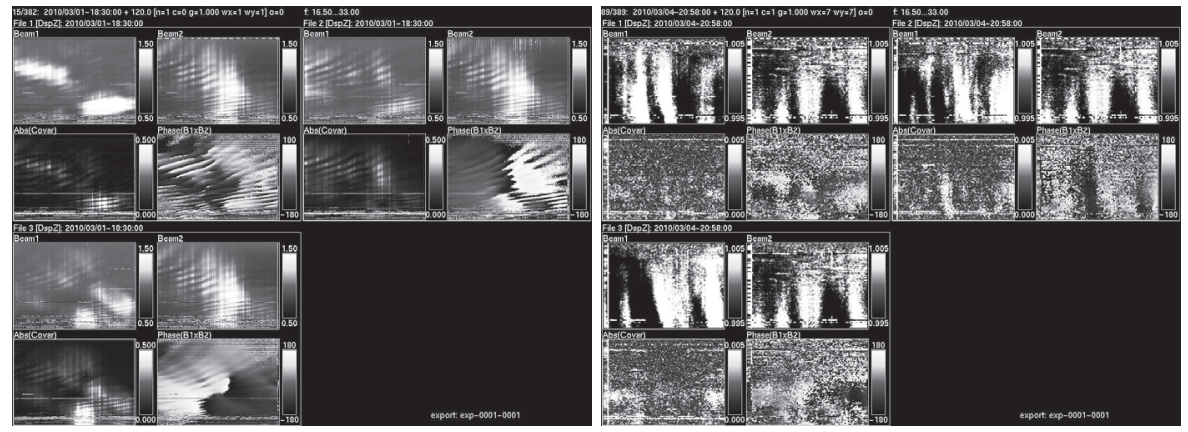

Figure 2: Dynamic spectra for typical IPS and non-typical ionospheric scintillations (left) and for an $A D$ Leo burst (right). For each figure, left two groups of 4 spectra correspond to two OFF channels; the right top group - to the ON channel. Horizontal axis corresponds to 2 minutes interval; the vertical one - to frequency range $16.5 \ldots 33.0 \mathrm{M} \mathrm{Hz}$. Each group of 4 spectra comprises the power for two antennas (top) and the amplitude and the phase of cross-correlation (bottom).

\subsection{Jupiter}

In the given investigations the best experimental parameters (sensitivity, dynamic range, resolution, analysis band, continuous observation duration) have been provided. This 
allows ones to study fine frequency-time features and modulation effects in the sporadic radio emission of Jupiter [Litvinenko et al., 2009]. In Fig. 3 (left) the dynamic spectrum shows a complex structure of its S-emission.
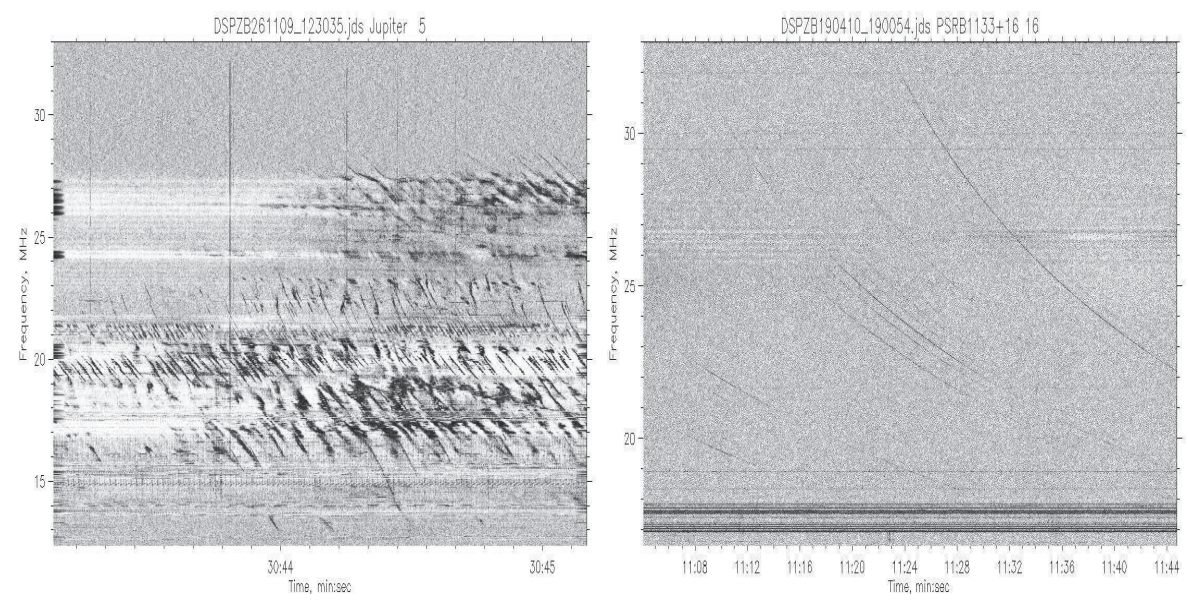

Figure 3: Dynamic spectra for Jupiter observation (left) and PSRB1133+16 (right). The left spectrum covers 2 seconds intervals (horizontal axis) and frequency range $12.5 \ldots 33.0 \mathrm{M} \mathrm{Hz}$; the right one - 40 minutes and $16.5 \ldots 33.0 \mathrm{M} \mathrm{Hz}$.

\subsection{Pulsars}

Pulsar radio emission is observed with UTR-2 during near 40 years already [Bruk, 2007; Ulyanov et al., 2006]. High sensitivity of equipments and methods provide opportunities to observe even individual pulses (Fig. 3). Considerable variations of pulse intensities are clearly seen. This demonstrates influences of interesting effects in the outer pulsar magnetosphere.

\subsection{Saturn Electrostatic Discharges}

SED radio emission has been detected with ground-based instrument (UTR-2) in 2006 after 30 year long attempts [Konovalenko et al., 2007]. This detection has become possible not only due to the high sensitivity of UTR-2 but also due to the data from space mission Cassini [Fischer et al., 2007] which have been used for the SED observations since 2004. Fig. 4 illustrates the outlook of one of the SED events which happened in the March 2010. The telescope was in ON-OFF mode: the main beam was directed to Saturn (ON mode), a secondary beam was shifted from Saturn by 1 degree (OFF mode). The SED manifests itself as a vertical spike allocated approx. $50 \mathrm{sec}(40 \%$ of the scan) from the beginning; the spike is clearly visible in ON channel and barely visible in OFF channel (as passed through a side-lobe of the secondary beam). 


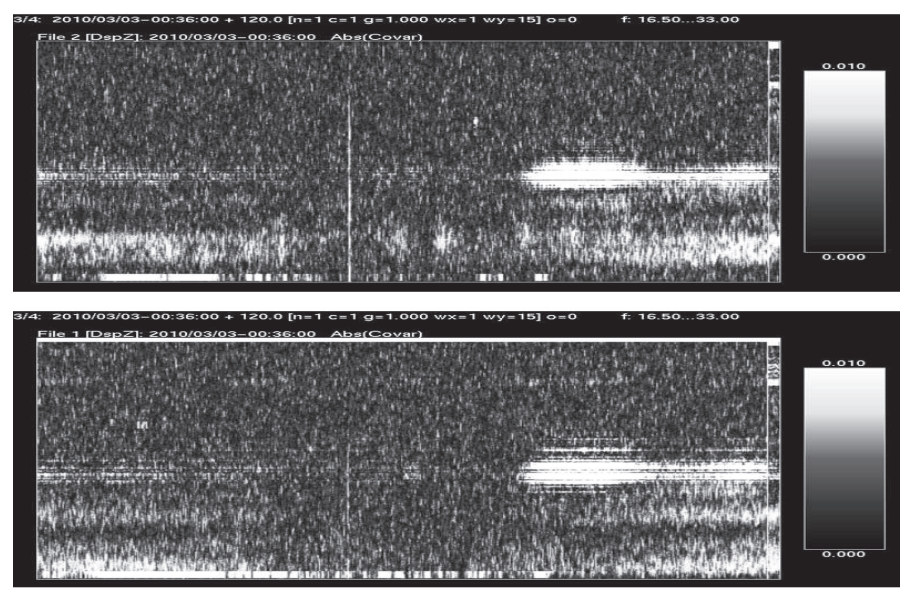

Figure 4: Saturn Electrostatic Discharges (SED) in ON-OFF mode of UTR-2. Dynamic spectra of the amplitude of cross-correlation are shown for 2 minutes intervals (horizontal axis) and frequency range 16.5 .. 33.0 $\mathrm{M} \mathrm{Hz}$ (vertical axis) for channel ON (top) and OFF (bottom).

\subsection{Regions of the Ionized Carbon}

The low frequency absorption recombination lines of carbon, which were detected more than 30 years ago at UTR-2, turned out to be effective means of the partially ionized cold interstellar medium diagnostics [Konovalenko and Stepkin, 2005]. For example, such medium can exist near B-type stars. Fig. 5 shows not-cleaned from interferences spectrum measured in the frequency band from 20 to $30 \mathrm{MHz}$ where it is possible to see numerous spectral features which looks as quasy-periodic negative spikes. It is possible to average them in order to achieve high sensitivity.

\section{7 $\quad$ Flare stars}

These objects are rather interestring for LF radio astronomy. An extensive observation campaign at UTR-2 began in 2007. An example of dynamic spectra for AD Leo are shown at Fig. 2 (right). A relatively large number of events like this one have already been registered. These findings point to high probability of detection of flares from stars at LF. Nevertheless, it is necessary to be cautious with final conclusions - because ionospheric scintillations and focusing of the radio sources of continuum located within the beam of the radio telescope are likely to have close resemblance to flares from stars. 


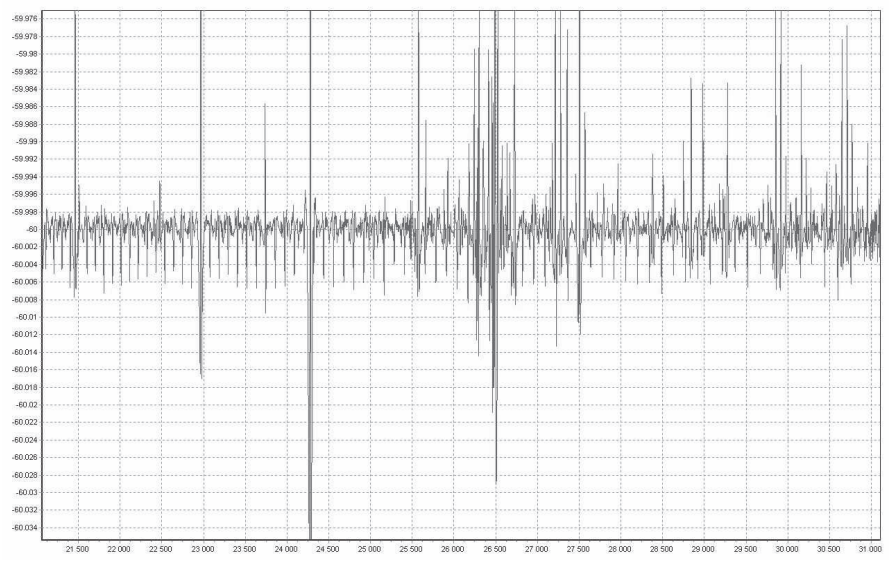

Figure 5: Low-frequency $(18 \ldots 32 \mathrm{MHz})$ absorption carbon radio recombination lines, $n=$ $600 \ldots 700$. The diagnostic of CII regions near B-stars, interplanetary plasma.

\section{Giant Ukrainian Radio Telescope (GURT) Concept}

At the present the experimental base of low-frequency radio astronomy successfully makes progress. Many states realize impressive scientific projects in this subject (see above). Notice that the current systems UTR-2, URAN remain indispensable and logically complementary for promising instruments, as they supply the frequency range less $30 \mathrm{MHz}$ up to the ionospheric cut-off $(10 \mathrm{MHz})$ together with the large effective area.

For a more effective development of low-frequency radio astronomy NASU started a special programm, devoted to a modernization of the present radio telescope (see above) as well as a creation of a new radio telescope GURT located on the UTR-2 radio telescope territory.

Main goal of GURT is high sensitive, broad-band, high time and frequency resolution, high interference immune studies of the Universe at extremely low frequencies when the sensitivity is not limited practically by the confusion effect $\left(\Delta S_{\operatorname{min.fl}} \leq \Delta S_{\text {min.conf. }}\right.$ ):

- frequency range (up to ionosphere limit) 10 - $70 \mathrm{MHz}$

- instant frequency band $60 \mathrm{MHz}$

- effective area ( step - by - step ) $10^{4} \ldots 10^{6}$ sq.m

- exceeding of antenna temperature $\geq 10 \mathrm{~dB}$

- exceeding of dynamic range $\geq 20 \mathrm{~dB}$

- two polarization, multi-beam capabilities 
- flexibility

- auto-spectra, cross-spectra, WFR (1 ms, $1 \mathrm{kHz})$

- complementary to UTR-2, URAN, LOFAR, LSS.

Fig. 6 (left) illustrates the external form of the dipole for the GURT. It has a wide range of frequencies $(10-80 \mathrm{MHz}$ ), high sensitivity (antenna temperature exceeding of galactic background over internal noises $\geq 10 \mathrm{~dB}$ ), Fig. 6 (right), high interference-immunity (output IP3 $\sim 47 \mathrm{dBm}$; output IP2 $86 \mathrm{dBm}, \mathrm{G} \sim 16 \mathrm{~dB}$ ) [Konovalenko et al., 2005]. This element is a basic part for building the 25-element subarray with an analog phasing. In Fig. 7 (left) the test results of such a subarray are shown in comparison with West-East antenna of the UTR-2 that confirm expected parameters. The next phasing of the GURT system, consisting on many subarrays, will be realized by digital methods. The outward appearance of the GURT part (6x25 elements) is represented in Fig7 (right).
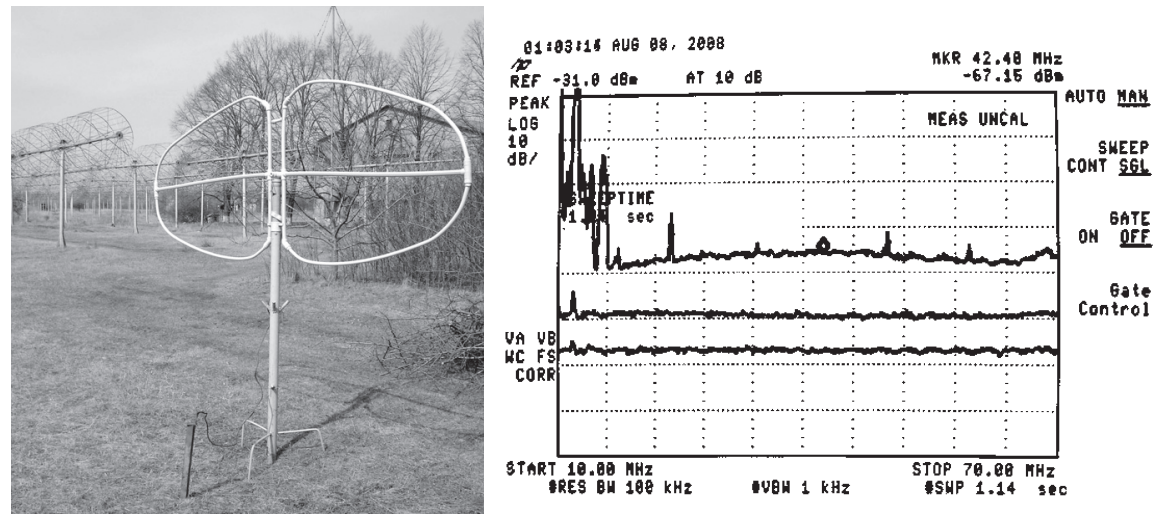

Figure 6: New broad-band, high sensitive, high dynamic range active antenna element for Giant Ukrainian Radio Telescope (left) and its frequency response of the dipole for the galactic background (right). The bottom line is the own noise of the amplifier, the middle one is the own noise of the preamplifier, and the top line is the response to the galactic background.

\section{Conclusion}

There is a broad field of activity in the planetary and stellar LF radio astronomy. The implementation of largest existing radio telescopes, the new generation high performance back-ends and methods gave many interesting astrophysical results. The creation and using of new generation low frequency radio telescopes (LOFAR, LWA, E-LOFAR, LSS, GURT, etc.) have good perspectives. It is also valuable for the experiments with the distant antennas (up to $1000 \mathrm{~km}$ ) in VLBI and/or synchronized modes. 

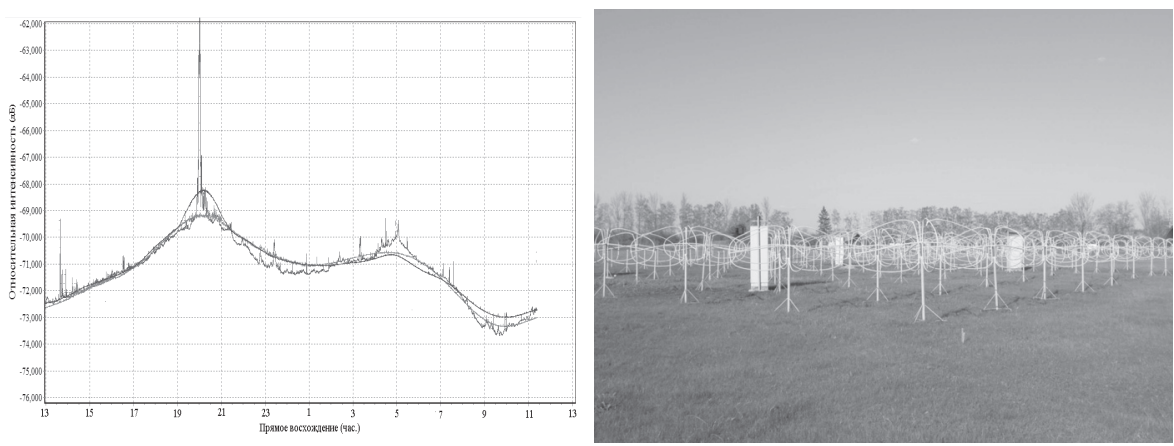

Figure 7: GURT array $6 \times 25$ elements (right) and the scans towards 3C405 by UTR-2 E-W and GURT sub-array (left): relative intensity (vertical axis, $d B$ ) against the right ascension (horizontal axis, hours).

\section{References}

Abranin, E. P., Yu. M. Bruk, V. V. Zakharenko, and A. A. Konovalenko, The new preamplification system for UTR-2 radio telescope, Experim. Astron. 11, 85-112, 2001.

Braude, S. Ya., A. V. Megn, and L. G. Sodin, The UTR-2 radio telescope at decameter wavelenghts, Antenna 26, 3-15, 1978.

Bruck, Yu. M., Decametric emission by Pulsars, Aust. J. Phys., 40, 861-870, 1987.

Fischer, G., W.S. Kurth, U. A. Dyudina, M. L. Kaiser, P. Zarka, A. Lecacheux, A. P. Ingersoll, and D. A. Gurnett, Analysis of a giant lighting storm on Saturn, Icarus, 190, 2, 528-544, 2007.

Konovalenko, A. A., Ukraine decameter wave radio astronomy systems and their perspectives, Geophys. Monogr., 119, 311-319, 2000.

Konovalenko, A. A. and S. V. Stepkin, Radio Recombination Lines, EAS Publication Series, 15, 271-295, 2005.

Konovalenko, A. A., I. S. Falkovich, N. N. Kalinichenko, A. A. Gridin, I. N. Bubnov, A. Lecacheux, C. Rosolen and H. O. Rucker, Thirty-Element Active Antenna Array as a Prototype of a Huge Low-Frequency Radio Telescope, Exp. Astron., 16, 3, 149-164, 2003.

Konovalenko, A. A., A. Lecacheux, H. O. Rucker, G. Fischer, E. P. Abranin, N. N. Kalinichenko, I. S. Falkovich, and K. M. Sidorchuk, Ground-based decameter wavelength observations of Saturn electrostatic discharges, Geophys. Res. Abs., 9, 04792, 2006.

Konovalenko, A. A., H. O. Rucker, A. Lecacheux, V. N. Melnik, I. S. Falkovich, N. N. Kalinichenko, M. R. Olyak, S. V. Stepkin, O. M. Ulyanov, A. V. Megn, S. L. Rashkovskij, V. A. Shepelev, B. Thide, Yu. V. Tokarev, A. I. Brazhenko, and V. V. Koshevoj, Utilizing existing decameter radio telescopes as pathfinders towards LOFAR-LWA-LOIS 
science and technology, in Planetary Radio Emission VI, edited by H. O. Rucker, W. S. Kurth, and G. Mann, Austrian Academy of Science Press, Vienna, 507-518, 2006 .

Konovalenko, A.A., A. A. Stanislavsky, E.P. Abranin, V. V. Dorovskij, V. N. Melnik, M. L. Kaiser, A. Lecacheux, and H. O. Rucker, Absorption in Burst Emission, Solar Phys., 245, 2, 345-354, 2007.

Kozhin, R. V., V.V. Vinogradov, and D. M. Vavriv, Low-noise, high dynamic range digital receiver/spectrometer for radio astronomy applications, MSMW Symp. Proc., Kharkov, Ukraine, 736-738, 2007.

Lecacheux, A., C. Rosolen, V. Clerc, P. Kleewein, H. O. Rucker, M. Y. Boudjada, and W. Van Diel, Digital techniques for ground-based low frequency radioastronomy, Proc. SPIE, 3357, 533-542, 1998.

Lecacheux, A., A.A. Konovalenko, and H. O. Rucker, Using large radio telescopes at decameter wavelengths, Planet. Space Sci., 52, 1357-1374, 2004.

Litvinenko, G. V., A. Lecacheux, H. O. Rucker, A. A. Konovalenko, B. P. Ryabov, U. Taubenschuss, V. V. Vinogradov, and V. E. Shaposhnikov, Modulation structures in the dynamic spectra of Jovian radio emission obtained with high time-frequency resolution, Astron. Astrophys., 493, 651-660, 2009.

Melnik, V. N., A. A. Konovalenko, H. O. Rucker, A. A. Stanislavsky, E. P. Abranin, A. Lecacheux, G. Mann, A. Warmuth , V.V. Zaitsev, M. Bonjada, V. V. Dorovskij., V. V. Zakharenko, V. N. Lisachenko, and C. Rosolen, Observations of solar type II bursts at frequencies 10-30 MHz, Solar Phys., 222, 151-166, 2004.

Ryabov, V. B., D. M. Vavriv, P. Zarka, B. P. Ryabov, R. Kozhin, V. V. Vinogradov, and L. Denis, A low-noise, high-dynamic-range, digital receiver for radio astronomy applications: an efficient solution for observing radio-bursts from Jupiter, the Sun, pulsars, and other astrophysical plasmas below $30 \mathrm{MHz}$, Astron. Astrophys., 510, $16-28,2010$.

Ulyanov, O. M., V. V. Zakharenko, A. A. Konovalenko, A. Lecacheux, C. Rosolen, and H. O. Rucker, Detection of individual pulses from pulsars B0809+74; B0834+06; B0950+08; B0943+10; B1133+16 in the decameter waves range, Rad. Phys. Rad. Astron., 11, 113-133, 2006. 
